\title{
ÍNDICES DE CONFIABILIDADE DA ANÁLISE DO ÂNGULO POPLÍTEO ATRAVÉS DA BIOFOTOGRAMETRIA
}

\author{
RELIABILITY INDEXES OF POPLITEAL ANGLE ANALYSIS THROUGH PHOTOGRAMMETRY \\ ÍNDICES DE FIABILIDAD DEL ANÁLISIS DEL ÁNGULO POPLITEO MEDIANTE FOTOGRAMETRÍA \\ ENSAIO CLÍNICO
}

Ricardo B. Duarte' (Fisioterapeuta) Sandro V. Romanatti ${ }^{1}$ (Fisioterapeuta) Hélcio Gongora' (Fisioterapeuta) Leandro Stetner Antonietti ${ }^{1}$ (Fisioterapeuta)

Leonardo Pires' (Fisioterapeuta) Moisés Cohen' (Ortopedista)

1. Centro de Traumatologia do Esporte, Departamento de Ortopedia e Traumatologia da Universidade Federal de São Paulo (Unifesp), São Paulo, SP, Brasil.

\section{Correspondência:}

Helcio Gongora. Rua Estado de Israel, 636. Vila Clementino, 04040-032, São Paulo, SP, Brasil. helciogongora@yahoo.com.br

\section{RESUMO}

Introdução: A avaliação e a mensuração objetiva da amplitude articular são de fundamental importância para o diagnóstico, planejamento e acompanhamento de um tratamento. Com o avanço da tecnologia, a biofotogrametria começa a ser utilizada com maior frequência no meio fisioterapêutico, mensurando a quantidade de movimento articular. O ângulo poplíteo foi escolhido por ser uma medida indireta da flexibilidade da musculatura ísquiossural. A retração dos isquiossurais pode resultar em problemas posturais significativos e produzir uma inclinação posterior contínua da pelve, afetando a marcha e o gesto esportivo, sendo também uma das causas de dores musculares ou articulares. Objetivos: Verificar as confiabilidades intra e inter-examinador da análise de ângulo poplíteo através a fotogrametria computadorizada, utilizando o software para avaliação postural (SAPo), buscando estabelecer a repetibilidade e a reprodutibilidade do método. Métodos: Foram avaliados 23 indivíduos (46 joelhos). Os voluntários foram posicionados em decúbito dorsal, com quadril e joelho do membro inferior avaliado fletidos a 90 graus, mantendo o membro contralateral em extensão. A seguir, o joelho do membro testado era estendido passivamente, até o ponto no qual se percebia primeira resistência dos músculos ao alongamento. Neste ponto, foi feito o registro do ângulo poplíteo. A análise estatística da confiabilidade inter e intra-examinador foi averiguada pelos resultados do coeficiente de correlação intra-classe (ICC) dos tipos 1,1 e 3,1 no teste de correlação de Pearson, considerando um nível de significância de $p<0,05$. Resultados: 0 método de avaliação do ângulo poplíteo pela biofotogrametria apresentou confiabilidade forte (ICC $=0,786$ ) intra-examinador e confiabilidade muito forte (ICC $=0,920)$ inter-examinador. Conclusão: A biofotogrametria é um método apropriado para a avaliação do ângulo poplíteo uma vez que os valores do coeficiente de correlação intra-classe intra e inter-examinadores atingiram níveis forte e muito forte, respectivamente, confirmando a reprodutibilidade e a repetibilidade desta técnica de avaliação.

Palavras-chave: amplitude de movimento articular, fotogrametria, avaliação.

\section{ABSTRACT}

Introduction: The assessment and objective measurement of joint range of motion are of fundamental importance for the diagnosis, planning and monitoring treatment. With the advancement of technology, photogrammetry is beginning to be used with greater frequency in the physical therapy practice, measuring the amount of joint motion. The popliteal angle was chosen because it provides an indirect measure of flexibility of the hamstring muscles. The retraction of the hamstrings can result in significant postural problems and produce a continuous posterior tilt of the pelvis, affecting the gait and sports movements, as well as causing muscle or joint pain. Objectives: To confirm the reliability intra- and inter-examiner analysis of popliteal angle through computerized photogrammetry using the Postural Assessment Software (SAPO), to establish the repeatability and reproducibility of the method. Methods: Twenty-three patients (46 knees) were evaluated. Volunteers were positioned supine with hip and knee of the evaluated leg flexed at 90 degrees, keeping the contralateral limb in extension. Next, the knee of the tested limb was passively extended until the point at which the first resistance to stretching was perceived. At this point, the popliteal angle was recorded. Statistical analysis of inter-and intra-examiner reliability was determined by the results of the coefficient of intra-class correlation (ICC) of types 1.1 and 3.1 in the Pearson correlation test, considering a significance level of $p<0.05$. Results: The measurement of the popliteal angle by photogrammetry showed strong intra-examiner reliability $(I C C=0.786)$ and very strong inter-examiner reliability $(I C C=0.920)$. Conclusion: The photogrammetry is an appropriate method for the evaluation of the popliteal angle since the coefficient of intra-class correlation and intra and inter-examiners reached strong and very strong levels, respectively, confirming the reproducibility and repeatability of this assessment technique.

Keywords: range of motion, articular, photogrammetry, evaluation.

\section{RESUMEN}

Introducción: La evaluación y la medición objetiva de la amplitud articular son de fundamental importancia para el diagnóstico, planificación y acompañamiento de un tratamiento. Con el avance de la tecnología, la fotogrametría está empezando a ser utilizada con mayor frecuencia en la práctica de fisioterapia para mensurar la cuantidad de 
movimiento articular. El ángulo poplíteo fue elegido porque proporciona una medida indirecta de la flexibilidad de la musculatura isquiotibial. La retracción de los isquiotibiales puede resultar en problemas de postura significativos y producir una inclinación posterior continua de la pelvis, afectando la marcha y el gesto deportivo, siendo también una de las causas de dolores musculares o articulares. Objetivos: Verificar la fiabilidad intra e inter-examinador de la análisis de ángulo poplíteo mediante fotogrametría computarizada, utilizando el software de evaluación postural (SAPO) para establecer la repetibilidad y la reproducibilidad del método. Métodos: Fueron evaluados 23 individuos (46 rodillas). Los voluntarios fueron posicionados en decúbito dorsal, con la cadera y la rodilla del miembro inferior evaluado flexionados a 90 grados, manteniendo el miembro contralateral en extensión. A continuación, la rodilla del miembro testado se extendió pasivamente hasta el punto en que se percibió la primera resistencia al estiramiento. En este punto, fue hecho el registro del ángulo poplíteo. El análisis estadístico de la fiabilidad inter e intra-examinador fue determinado por los resultados del coeficiente de correlación intra-clase (ICC) de los tipos 1.1 y 3.1 en la prueba de correlación de Pearson, considerando un nivel de significación de $p<0,05$. Resultados: La medición del ángulo poplíteo por fotogrametría mostró fuerte fiabilidad intra-examinador (ICC $=0,786)$ y muy fuerte fiabilidad inter-examinador (ICC = 0,920). Conclusión: La fotogrametría es un método apropiado para evaluación del ángulo poplíteo una vez que los valores del coeficiente de correlación intra-clase, intra einter-examinador llegaron a niveles fuerte y muy fuerte, respectivamente, confirmando la reproducibilidad y la repetibilidad de esta técnica de evaluación.

Palabras clave: amplitud de movimiento articular, fotogrametría, evaluación.

\section{INTRODUÇÃO}

A avaliação postural é a relação cinemática entre as posições dos complexos articulares do corpo em um dado momento. Em um alinhamento ideal, espera-se que os músculos, articulações e suas estruturas esqueléticas encontrem-se em estado de equilíbrio dinâmico gerando uma quantidade mínima de esforço e sobrecarga, conduzindo a uma eficiência ótima para o aparelho locomotor ${ }^{1}$. A avaliação e a mensuração objetiva da amplitude articular são de fundamental importância para o diagnóstico, planejamento do tratamento fisioterapêutico e acompanhamento dos resultados do mesmo².

Esse recurso é de fundamental importância para detectar as mudanças no comprimento e na extensibilidade muscular, que são as maiores causas das disfunções do movimento ${ }^{3}$. O encurtamento ou retração refere-se à redução do comprimento de uma unidade musculotendínea que permanece saudável, resultando em limitação da amplitude articular ${ }^{4}$.

Em particular, o complexo muscular isquiossural corresponde aos músculos: semimenbranoso, semitendinoso e bíceps femoral ${ }^{4}$. Este grupo muscular é composto por estruturas biarticulares responsáveis pela extensão de quadril e flexão do joelho, simultaneamente ${ }^{4,5}$. A retração dos isquiossurais pode resultar em problemas posturais significativos, e produzir uma inclinação posterior contínua da pelve, afetando a marcha e o gesto esportivo, sendo a causa de dores musculares ou articulares nos membros inferiores com seu consequente desalinhamento ${ }^{4,6}$.

Distúrbios que cursam com dor no joelho podem estar associados a alterações da musculatura posterior da coxa, com reflexo direto no movimento de extensão do joelho. A dor anterior está relacionada, na maioria das vezes, a encurtamentos musculares das extremidades inferiores $^{7}$. O método mais utilizado para avaliar a retração da musculatura isquiossural é a mensuração do ângulo poplíteo ${ }^{8,9}$.

O ângulo poplíteo mede indiretamente o comprimento dos músculos isquiossurais. Este ângulo também é utilizado para determinação da idade gestacional em neonatos e na propedêutica de doenças, entre elas a doença de Scheuermann, a doença de Osgood-Schlater, a síndrome de Larsen e as doenças neuromusculares s,10. $^{9}$.

A goniometria manual é um método largamente utilizado clinicamente para avaliação deste ângulo ${ }^{11-13}$. Entre as suas vantagens estão o baixo custo do instrumento e a fácil mensuração, que depende quase que exclusivamente da experiência anterior do avaliador ${ }^{11}$.
No entanto, com o avanço da tecnologia, a fotogrametria digital tem sido considerada uma alternativa para a avaliação quantitativa dos ângulos posturais, podendo ser utilizada para a realização de medidas lineares e angulares ${ }^{14,15}$. Ela possibilita o registro de mudanças sutis e da inter-relação entre partes diferentes do corpo humano difíceis de serem mensuradas ou registradas por outros meios ${ }^{14}$.

Na fotogrametria existe a facilitação no processo de arquivamento e possibilidade de conjugação a processos computadorizados de mensuração, tendo como resultado a fotogrametria computadorizada. Portanto, a fotogrametria computadorizada é a combinação da fotogrametria digital com softwares que permitem a mensuração de ângulos e distâncias horizontais e verticais para finalidades diversas ${ }^{2,16}$.

A utilização da fotogrametria pode facilitar a quantificação das variáveis morfológicas relacionadas à postura, trazendo dados mais confiáveis do que os obtidos pela observação visual. Fato importante, tanto para a credibilidade da fisioterapia clínica, quanto para a confiabilidade das pesquisas em reabilitação ${ }^{2,15}$.

Na literatura consultada, no entanto, não foram encontrados trabaIhos que realizassem a análise de reprodutibilidade e repetibilidade da mensuração do ângulo poplíteo pela biofotogrametria, especificamente.

O objetivo deste estudo foi verificar as confiabilidades intra e inter-examinador da análise de ângulo poplíteo por meio da fotogrametria computadorizada, utilizando o software para avaliação postural (SAPo), buscando estabelecer, desta forma, a repetibilidade e a reprodutibilidade do método.

\section{MATERIAIS E MÉTODOS}

Foram avaliados 23 indivíduos (46 joelhos), escolhidos de maneira aleatória dentre uma população composta por atletas e frequentadores do Centro Olímpico de Treinamento e Pesquisa de São Paulo, SP, Brasil (COTP-SP), dentro dos seguintes critérios: (1) ausência de dor em membros inferiores e coluna lombar nos últimos três meses; (2) ausência de alteração nos treinamentos, referente à intensidade, volume e frequência nas últimas três semanas, no caso de atletas; (3) ausência de doenças sistêmicas ou neurológicas e lesões ou deformidades musculoesqueléticas evidentes na inspeção e na avaliação subjetiva.

Todos os voluntários receberam informações para participação do projeto e assinaram um termo de consentimento formal, concordando em participar da pesquisa, de acordo com a resolução 196/96 do Conselho Nacional de Saúde. O protocolo experimental deste estudo 
foi aprovado pelo Comitê de Ética e Pesquisa da Universidade Federal de São Paulo (UNIFESP)., SP, Brasil.

Os registros fotográficos foram realizados com uma câmera digital (NIKON ${ }^{\circledR 200)}$ posicionada paralelamente ao chão, sobre um tripé nivelado a 0,8 m de altura. $\mathrm{O}$ ambiente era bem iluminado com fundo não reflexivo e reservado, permitindo a privacidade do sujeito a ser fotografado. As imagens digitais obtidas com resolução de 4.2 megapixels foram armazenadas para posterior análise. Todas as fotografias foram realizadas por um único fotógrafo, respeitando o mesmo horário de coleta para a primeira e segunda séries. Os marcadores foram sempre posicionados pelo mesmo experimentador. Em cada série, foram realizadas duas fotografias para cada examinador (uma para cada joelho).

Para o registro fotográfico, os participantes permaneceram em decúbito dorsal horizontal em uma maca de $77 \mathrm{~cm}$ de altura, $60 \mathrm{~cm}$ de largura e 1,8 m de comprimento. A câmera permaneceu a $3 \mathrm{~m}$ de distância da maca, em um tripé nivelado a 0,88 m de altura. A técnica de avaliação do ângulo poplíteo foi a descrita por Vernieri ${ }^{10}$.

O quadril e o joelho do membro inferior testado foram fletidos a 90 graus, mantendo o membro contralateral em extensão completa sobre a maca, não sendo permitida a flexão ativa do quadril ou joelho. Para assegurar a flexão do quadril em 90 graus foi utilizada uma faixa ajustável de $5 \mathrm{~cm}$ de largura e $5 \mathrm{~m}$ de comprimento, presa à cabeceira da maca em uma haste perpendicular, passando pela região poplítea do membro inferior a ser testado. Apesar da faixa não ser descrita na literatura, a mesma não alterou o método de Vernieri, apenas sendo utilizada com o objetivo de melhorar a visualização dos pontos na fotografia a ser analisada. A seguir, o joelho do membro testado foi estendido passivamente, até o ponto no qual se percebia primeira resistência dos músculos ao alongamento. Neste ponto, foi feito o registro do ângulo poplíteo (figura 1).

As marcações foram realizadas com etiquetas adesivas verdes de 0,9 mm de diâmetro para os pontos a serem visualizados no plano sagital. Os seguintes pontos anatômicos foram utilizados: grande trocânter, cabeça da fíbula e maléolo lateral. Estes pontos foram utilizados por terem sido sugeridos em estudos prévios ${ }^{2,15}$.

Os registros fotográficos foram analisados por meio do programa SAPO. O programa permite traçar digitalmente as retas que determinaram os valores angulares em graus para os pontos de referência correspondentes.

As medidas angulares para a avaliação inter-examinador foram obtidas em um único dia, dos registros da primeira série, por dois examinadores fisioterapeutas previamente treinados para utilizar o programa e sem conhecimento mútuo dos valores anotados, verificando assim, a reprodutibilidade do método.

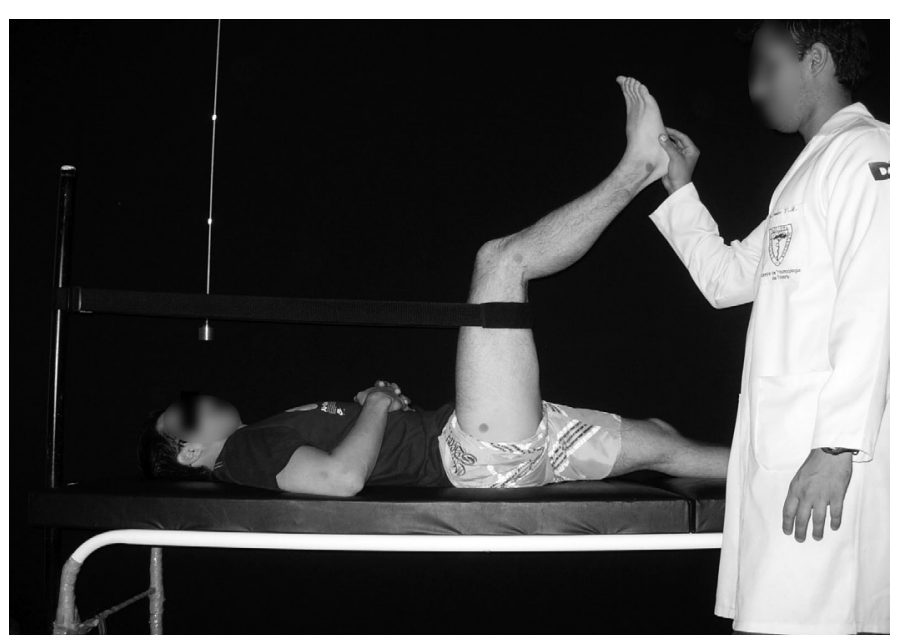

Figura 1. Posicionamento para o registro fotográfico.
As medidas angulares para a avaliação intra-examinador foram obtidas por um único examinador em duas ocasiões, espaçadas por 1 semana, comparando assim, a repetibilidade do método.

\section{Análise estatística}

Os dados foram expostos nos planos tabular e gráfico.

A normalidade dos dados foi verificada com o teste de Shapiro-Wilk, sendo que todos apresentaram distribuição normal.

A estatística descritiva constou do cálculo de média, desvio-padrão, mínimo e máximo para todos os dados contínuos e discretos e frequências absoluta e relativa para os dados categóricos.

Para o cálculo da confiabilidade intra e inter-examinador utilizou-se o coeficiente de correlação intraclasse (ICC - Intra-class correlation coeficient) dos tipos 3,1 e 1,1, segundo Yaffe ${ }^{17}$. Considerou-se como aceitável a confiabilidade cujo valor de ICC estivesse acima de 0,40, com nível de significância $\leq 0,05$ e com valor de $p$ do teste do sinal $\leq 0,05^{18}$.

Para classificação do ICC foi assumido o valor $\geq 0,80$ como excelente nível de confiabilidade, como sugerido por Yaffe ${ }^{17}$. Os outros intervalos de confiabilidade foram adaptados conforme sugerido por Fleiss (apud Delitto \& Strube) ${ }^{19}$ : valores $<0,40$ têm uma confiabilidade pobre; e entre 0,40 e 0,79 têm uma boa confiabilidade.

Calculou-se também a correlação de Pearson e a diferença estatística entre os valores: na análise intra-examinador com o teste $t$ de Student para variáveis dependentes e na análise inter-examinador com o teste $t$ de Student para variáveis independentes (foi aplicado o teste de Levene e como não houve diferença estatisticamente significante entre os coeficientes de variação não foi utilizada a correção de Welch) 18,20,21.

Para as correlações foram consideradas: $|0,00|$ a $|0,19|$ - correlação bem fraca; $|0,20|$ a $|0,39|$ - correlação fraca; $|0,40|$ a $|0,69|$ - correlação moderada; $|0,70|$ a $|0,89|$ - correlação forte; $|0,90|$ a $|1,00|$ - correlação muito forte. Valores estes considerados somente quando o nível de significância encontrado foi $\leq 0,05$.

O erro-padrão (EP) foi calculado para cada correlação e foi expressa em unidades de medida. A equação para o cálculo do EP foi a seguinte:

$E P=D P \times \sqrt{ }(1-I C C)$, onde DP é o desvio-padrão e ICC o coeficiente de correlação interclasse (Hayes et al. $)^{22}$.

Foi assumido valor de $p \leq 0,05$ ( $\alpha=5 \%$ ) como estatisticamente significante.

Para as análises foram utilizados os softwares estatísticos Prism 5.0 for Windows (GraphPad Software, Inc.) e SPSS Statistics 17.0 for Windows (SPSS, Inc.) $)^{21,23}$.

\section{RESULTADOS}

A amostra constou de 23 indivíduos (46 joelhos), dentro da qual, a sua caracterização foi realizada baseada em alguns dados antropométricos.

A média de idade dos indivíduos foi de 23,78 anos (mínimo de 18 anos, máximo de 28 anos); a divisão entre os gêneros foi de 47,8\% de mulheres (11 indivíduos) e 52,2\% de homens (12 indivíduos); a altura média foi de $172 \mathrm{~cm}$ (mínimo de $158 \mathrm{~cm}$, máximo de $186 \mathrm{~cm}$ ); a média de massa corporal foi de 70,35 kg (mínimo de 49 Kg, máximo de 96 kg), e a média do Índice de Massa Corporal (IMC) foi de 23,79 kg/m² (mínimo de $20,07 \mathrm{~kg} / \mathrm{m}^{2}$, máximo de $29,96 \mathrm{~kg} / \mathrm{m}^{2}$ ). Entre os indivíduos testados 91,3\% (21 indivíduos) tinham dominância do membro inferior direito e 8,7\% (2 indivíduos) tinham dominância do membro inferior esquerdo.

A tabela 1 mostra os dados de média e desvio padrão da primeira avaliação (D1) dos dois avaliadores (A e B) e da segunda avaliação (D2) dos dois avaliadores (A e B).

A tabela 2 mostra os valores da diferença estatística, além do índice de correlação de Pearson, dos valores de ICC e erro padrão dos dados referentes às análises intra- e inter-examinadores. 
Tabela 1. Média e desvio padrão dos valores de ângulo poplíteo na primeira (D1) e segunda (D2) avaliações.

\begin{tabular}{c|c|c|c|c|c}
\hline & N & Mínimo & Máximo & Média & Desvio Padrão \\
\hline $\begin{array}{l}\text { D1 Avaliador A } \\
\text { Membro Inf E }\end{array}$ & 23 & 121,20 & 173,60 & 139,5696 & 12,88521 \\
\hline $\begin{array}{l}\text { D1 Avaliador A } \\
\text { Membro Inf D }\end{array}$ & 23 & 123,70 & 175,70 & 141,8087 & 13,40536 \\
\hline $\begin{array}{l}\text { D1 Avaliador B } \\
\text { Membro Inf E }\end{array}$ & 23 & 127,20 & 176,60 & 144,8565 & 12,77337 \\
\hline $\begin{array}{l}\text { D1 Avaliador B } \\
\text { Membro Inf D }\end{array}$ & 23 & 125,50 & 166,80 & 144,6391 & 11,65975 \\
\hline $\begin{array}{l}\text { D2 Avaliador A } \\
\text { Membro Inf E }\end{array}$ & 23 & 120,20 & 146,30 & 131,8000 & 8,33165 \\
\hline $\begin{array}{l}\text { D2 Avaliador A } \\
\text { Membro Inf D }\end{array}$ & 23 & 117,20 & 157,00 & 133,0304 & 10,20122 \\
\hline $\begin{array}{l}\text { D2 Avaliador B } \\
\text { Membro Inf E }\end{array}$ & 23 & 120,40 & 145,00 & 132,1087 & 7,74602 \\
\hline $\begin{array}{l}\text { D2 Avaliador B } \\
\text { Membro Inf D }\end{array}$ & 23 & 111,40 & 156,50 & 134,9739 & 11,24650 \\
\hline
\end{tabular}

Tabela 2. Diferença estatística referente às análises intra e inter-examinadores.

\begin{tabular}{c|c|c}
\hline & \multicolumn{2}{|c}{ Biofotogrametria } \\
\hline Correlação de Pearson $(r-p)$ & $r=0,676-p<0,001$ & $r=0,852-p<0,001$ \\
\hline Valor de $p$ do teste $t$ de Student & $<0,001^{a}$ & $0,149^{b}$ \\
\hline ICC & 0,786 & 0,920 \\
\hline Valor de $p$ do ICC & $<0,001$ & $<0,001$ \\
\hline IC 95\% do ICC (superior - inferior) & $0,676-0,858$ & $0,879-0,947$ \\
\hline Valor de p do teste do sinal & $<0,001$ & 0,005 \\
\hline Erro-padrão & 5,62 & 3,44 \\
\hline
\end{tabular}

a: Teste $t$ de Student para amostras dependentes; b: Teste $t$ de Student para amostras independentes.

\section{DISCUSSÃO}

No estudo da mensuração da amplitude de movimento, observa-se a goniometria como padrão ouro, pelo seu baixo custo, simples manuseio, e confiabilidade de boa a excelente ${ }^{2}$. No entanto, com o avanço da tecnologia, a biofotogrametria começa a ser utilizada com maior frequência no ambiente clínico.

No presente estudo a biofotogrametria foi utilizada na avaliação do encurtamento da musculatura isquiossural. A medição da flexibilidade isquiossural pode ser realizada através de diversas técnicas, tais como: teste da elevação da perna estendida descrito por Kendall'; teste sente alcance com bloco de Wells descrito por Polock \& Wilmore, ${ }^{24}$ e Baltaci et al. ${ }^{25}$; e a técnica adotada em nossa metodologia, a medida do ângulo poplíteo descrita por Vernieri ${ }^{4}$.

O teste do ângulo poplíteo foi descrito originalmente por Amiel-Tison ${ }^{26}$ em 1968 que media o ângulo formado na região posterior do joelho. Bleck ${ }^{27}$ em 1979 chamou de ângulo poplíteo aquele formado pelo eixo longitudinal da perna e o prolongamento do eixo longitudinal da coxa na região anterior do joelho. Vernieri ${ }^{4}$ definiu o ângulo poplíteo como o ângulo formado pelo eixo do fêmur e da tíbia, estando a coxo-femoral em flexão de $90^{\circ}$, a contra lateral em extensão máxima possível e o paciente em decúbito dorsal, medindo o ângulo formado na face posterior do joeIho. O valor do ângulo, desta forma, será diretamente proporcional ao comprimento muscular e inversamente proporcional ao seu encurtamento ${ }^{28}$.

Outro ponto passível de discussão é o momento da extensão do joelho no qual é registrada a medida do ângulo. A mensuração descrita por Vernieri considera a primeira resistência à tensão aplicada à musculatura posterior da coxa, esse achado foi a base para outros artigos encontrados ${ }^{4,10,28}$. Buscando uma padronização da técnica, acreditamos ser este o ponto de extensão mais indicado para a medição do ângulo poplíteo e, por essa razão, foi utilizado em nossa metodologia.
Os resultados desta pesquisa mostraram que o método proposto para avaliação do ângulo poplíteo pela biofotogrametria apresentou confiabilidade forte (ICC =0,786) intra-examinador e confiabilidade muito forte (ICC =0,920) inter-examinador.

Segundo lunes et al. ${ }^{15}$, a fotogrametria computadorizada na avaliação postural apresentou confiabilidade forte (ICC entre 0,71 e 0,79) intra e inter-examinadores para a maioria das medidas angulares avaliadas (dentre as quais não estava o ângulo poplíteo), no entanto com baixa repetibilidade. Os mesmos resultados foram encontrados por Zonnenberg et al. ${ }^{29}$, que encontraram confiabilidade intra e inter-examinadores muito forte para todas as medidas angulares realizadas pela fotogrametria, porém, com baixa repetibilidade do método. Nesses trabalhos o ICC intra-examinador foi calculado através de medidas obtidas, por um único examinador, em duas ocasiões diferentes, no entanto, eram avaliadas apenas as fotografias da primeira série com um intervalo mínimo de quatro semanas e, sua repetibilidade foi calculada a partir das medidas obtidas, por um único examinador, comparando os registros da primeira e segunda séries.

Já Braun e Amundson ${ }^{30}$ encontraram confiabilidade e repetibilidade adequadas para a avaliação postural de cabeça e ombros. Outros estudos, como os de Sato et al. ${ }^{31}$ e Hayes et al. ${ }^{22}$ também apresentaram alta confiabilidade de técnicas fotogramétricas para a avaliação da amplitude de movimento de ombro e tronco.

O trabalho de Amorim et al. ${ }^{32}$ foi o único encontrado relacionando a biofotogrametria e a mensuração do ângulo poplíteo. A pesquisa consistia na comparação da fotogrametria computadorizada e a goniometria. Os resultados mostraram que não havia diferença estatisticamente significativa entre os grupos da goniometria e da fotogrametria, isto é, as medidas entre os dois grupos foram semelhantes. Desta forma, os autores validaram o método, no entanto, não realizaram a confiabilidade intra- e inter-examinadores, proposta em nosso trabalho. É importante ressaltar que este estudo utilizou o programa AutoCAD $2000^{\circledR}$, um software pago, e nosso estudo se utilizou de um software gratuito, o SAPo, e extremamente confiável para esta avaliação, constituindo um custo a menos.

Nos estudos que utilizaram a biofotogrametria, os examinadores avaliaram a mesma foto em duas situações diferentes ${ }^{15,30}$. Um diferencial do presente trabalho foi a realização de duas fotografias em duas situações, sendo avaliadas por dois examinadores diferentes, com cada examinador avaliando suas fotografias. Essa metodologia foi adotada buscando reproduzir ao máximo a realidade clinica, na qual um mesmo fisioterapeuta faz o registro fotográfico e o reavalia. Dentro das condições testadas, a fotogrametria computadorizada apresentou valores altos de confiabilidade, demonstrando ser um método eficaz na avaliação do ângulo poplíteo, sendo porém, necessário ainda ser testado para diferentes movimentos em diferentes articulações, com diferentes softwares e diferentes condições de imagem.

As limitações do estudo foram: o baixo número de indivíduos da amostra e a subjetividade quanto à determinação da primeira resistência da musculatura isquiossural, definida por Vernieri ${ }^{10}$ para o estabelecimento do ângulo poplíteo. Além disso, apesar do baixo custo do processo, a fotogrametria ainda é mais dispendiosa que a goniometria como método de avaliação de ângulos.

O presente estudo demonstrou que o método de avaliação do ângulo poplíteo através da biofotogrametria apresentou confiabilidade intra-examinador classificada como boa nos critérios adotados, comprovando a repetibilidade do método, e confiablilidade inter-examinador classificada como excelente dentro dos critérios adotados, comprovando a reprodutibilidade do método. No entanto, mais estudos são 
necessários de forma a compreender as alterações do ângulo poplíteo dentro do contexto de cada patologia especificamente.

\section{CONCLUSÃO}

De acordo com os objetivos determinados pela pesquisa, conclui-se que a biofotogrametria é um método apropriado para a avaliação do ângulo poplíteo dentro das condições estabelecidas, devido aos valores estatísticos encontrados que confirmam a reprodutibilidade e a repetibilidade da técnica, por seus índices inter- e intra-examinadores terem sido classificados com confiabilidade de forte a muito forte. Desta forma, revelando-se um método confiável para a avaliação clínica atual.

Todos os autores declararam não haver qualquer potencial conflito de interesses referente a este artigo.

\section{REFERÊNCIAS}

1. Kendall FP, McCreary EK, Provance PG. Músculos - Provas e funçōes. 4a Ed. São Paulo: Editora Manole; 1995.

2. Sacco ICN, Albert S, Queiroz BWC, Pripas D, Kieling I, Kimura AA, Sellmer AE, Malvestio RA, Será MT. Confiabilidade da fotogrametria em relação à goniometria para avaliação postural de membros inferiores. Rev Bras Fisioter. 2007;11(5):411-7.

3. Lima RCM, Pessoa BF, Martins BLT, De Freitas DBN. Análise da durabilidade do efeito do alongamento muscular dos isquiotibiais. Acta Fisiatr. 2006;13(1):32-8.

4. Polachini LO, Fusazaki L, Tamaso M, Tellini GG, Masiero D. Estudo comparativo entre três métodos de avaliação do encurtamento de musculatura posterior de coxa. Rev Bras Fisioter. 2005;9(2):187-93.

5. Noyes FR, Sonstegard DA. Biomechanical function of the pés anserinus at the knee and the effect of its transplantation. J Bone Joint Surg Am. 1973;35[A]:1225-40.

6. Calliet R. Exame do paciente com dor lombar. In: Síndrome da dor lombar. Porto Alegre: Artmed; 2001. p.97-137.

7. Shrier I, Ehrmann-Feldman D, Rossingnol M, Abenhaim L. Risk factors for development of lower limb pain in adolescents. J Reumathol 2001;28(3):604-9.

8. Forlin E, Andújar AL, Alessi S. Padrões de normalidade do exame físico dos membros inferiores em crianças na idade escolar. Rev Bras Ortop. 1994;29(8):601-7.

9. Malheiros DS, Cunha FM, Lima CFLA. Análise da medida do ângulo poplíteo em crianças de sete a treze anos de idade. Rev Bras Ortop. 1995;30(9):693-8.

10. Pereira LF. Ângulo poplíteo: Avaliação em 590 crianças de 6 a 14 anos. Trabalho de conclusão apresentado à Universidade Federal de Santa Catarina. 2004.

11. Venturini C, André A, Aguilar BP, Giacomelli B. Confiabilidade de dois métodos de avaliação da amplitude de movimento ativa de dorsiflexão do tornozelo em indivíduos saudáveis. Acta Fisiatr. 2006;13(1):41-5.

12. Brosseau L, Tousignant M, Budd J, Chartier N, Duciaume L, Plamondon S, et al. Intratester and intertester reliability and criterion validity of the parallelogram and universal goniometers for active knee flexion in healthy subjects. Physiother Res Int. 1997;2(3):150-66.

13. Sabari JS, Maltzev I, Lubarsky D, Liszkay E, Homel R. Goniometric assessment of shoulder range of motion: comparison testing in supine and sitting positions. Arch Phys Med Rehabil. 1998;79:647-51.

14. ASPRS - American Society for Photogrammetry and Remote Sensing. What is ASPRS - definition [homepage na Internet]. Bethesda American Society for Photogrammetry and Remote Sensing: 2000 [atualizada em 2006 Nov 16; acesso em 2006 Out 24]. Disponível em: http://www.asprs.org/ society/about.html.

15. Iunes DH, Castro FA, Salgado HS, Moura IC, Oliveira AS, Bevilaqua-Grossi D. Confiabilidade intra e interexaminadores e repetibilidade da avaliação postural pela fotogrametria. Rev Bras Fisioter. 2005;9(3):327-34
16. Watson AWS, Macdonncha C. A reliable technique for the assessment of posture: assessment criteria for aspects of posture. J Sports Med Phys Fitness. 2000; 40(3):260-70.

17. Yafee RA. Enhancement of reliability analysis: application of intraclass correlations with SPSS/Windows v.8; available: www.nyu.edu/acf/socsci/Docs/intracls.html. 1998.

18. Dytham C. Choosing and using statistics: a biologist's guide Calvin Dytham. Oxford: Blackwell Science, 1999. 218p.

19. Fleiss JL, 1986 apud Delitto A, Strube MJ. Reliability in the clinical setting. American Physical Therapy Association Research Section Newsletter. 1991;24:2-8.

20. Shimakura SE. Silvia Emiko Shimakura: Laboratório de Estatística e Geoinformação, UFPR, Brasil, jan 2007. Disponível em: <http://leg.ufpr.br/ shimakur/>. Acesso em: 15 nov. 2007.

21. Vieira S. Bioestatística: Tópicos Avançados. 2a Ed. Rio de Janeiro: Campus, 2003. 216p.

22. Hayes K, Walton JR, Szomor ZL, Murrel GAC. Reliability of five methods for assessing shoulder range of motion. Aust J Physiother. 2001;47: 89-94.

23. Graphpad Software. GraphPad Prism 5.0: Learning Guide. Disponível em: <http;//www.graphpad.com/ help/Prism5/Prism5Help.html>. Acesso em 20 jan 2008.

24. Pollock ML, Wilmore JH. Selecionamento clínico e metodologia de avaliação, prescrição de programas de prevenção e reabilitação. In: Exercícios na saúde e na doença. Rio de Janeiro: MEDSi. 1993. p.340-362.

25. Baltaci G, Um N, Besler A, Gerçeker S. Comparison of three different sit and reach tests for measurement of hamstring flexibility in female university studentes. Br J Sports Med. 2003;37:59-61

26. Amiel-Tison C. Neurological evaluation of the maturity of newborn infants. Arch Dis Child. 1968;43:89-93.

27. Bleck EE. Orthopaedic assessment. In: Orthopaedic management of cerebral palsy. Philadelphia, W.B. Saunders, 29-33, 1979.

28. Afonso Filho A, Navarro RD. Avaliação do ângulo poplíteo em joelhos de adolescentes assintomáticos Rev Bras Ortop. 2002;37(10):461-6.

29. Zonnenberg AJ, Maanem V, Elvers JWH, Oostendorp RAB. Intra/interrater reliability of measurements on body posture photographs. J Cranomand Pract. 1996;14(4):326-31.

30. Braun BL, Amundson LR. Quantitative assessment of head and shoulder posture. Arch Med Phys Rehabil. 1989;70(4):322-9.

31. Sato TO, Vieira ER, Coury HG. Análise da confiabilidade de técnicas fotogramétricas para medir a flexão anterior do tronco. Rev Bras Fisioter. 2003;7(1):53-99.

32. Amorim DA, Ribeiro EM, Cordeiro GG, Silva MAS. O programa AutoCad $2000^{\circledR}$ como forma de medida angular para articulações. Trabalho de conclusão apresentado à Faculdade de Fisioterapia da Universidade de Itaúna. 2005. Disponível em: <http://www.wgate.com.br/conteudo/medicinaesaude/ fisioterapia/variedades/analise_autocad/analise_autocad.htm>. Acessado em 28 jan 2009. 